\title{
Development of a novel 3D-printed multifunctional thorax model simulator for the simulation-based training of tube thoracostomy
}

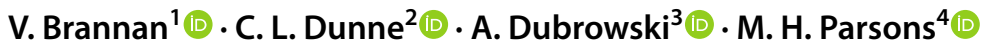

Received: 24 September 2020 / Accepted: 9 February 2021 / Published online: 30 March 2021

() The Author(s), under exclusive licence to Canadian Association of Emergency Physicians (CAEP)/ Association Canadienne de Médecine d'Urgence (ACMU) 2021

\begin{abstract}
Tube thoracostomy is a high-acuity, low-occurrence (HALO) procedure with significant morbidity when performed incorrectly; this is amendable through simulation. Commercially available trainers exist but often have limited realism or exorbitant cost. Three-dimensional (3D) printing produces realistic and cost-effective models suitable for simulation, but no simulator has been developed for tube thoracostomy. The aim of this paper is to describe the initial development of a multifunctional 3D-printed thorax trainer for the instruction of tube thoracostomy. The thorax model was developed in conjunction with a multi-disciplinary team using 3D-printing capable software. An existing ribcage model was modified and printed in separate elements, including bony portions (ribcage, sternum and clavicles), flexible joints, skin, heart and lungs and then assembled. The total printing cost was $\$ 180$ CAD. Future research will focus on incorporating the model's ability to simulate other HALO procedures and evaluating it as a training adjunct.
\end{abstract}

Keywords Emergency Medicine $\cdot 3$ D-printing $\cdot$ simulation $\cdot$ education

\section{Résumé}

La thoracostomie par tube est une procédure de haute acuité et de faible occurrence (HALO) avec une morbidité significative lorsqu'elle est mal exécutée; ceci est modifiable par simulation. Il existe des formateurs disponibles dans le commerce, mais ils sont souvent d'un réalisme limité ou d'un coût exorbitant. L'impression tridimensionnelle (3D) produit des modèles réalistes et rentables adaptés à la simulation, mais aucun simulateur n'a été développé pour la thoracostomie tubulaire. L'objectif de ce document est de décrire le développement initial d'un simulateur de thorax multifonctionnel imprimé en 3D pour l'enseignement de la thoracostomie tubulaire. Le modèle du thorax a été développé en collaboration avec une équipe pluridisciplinaire utilisant un logiciel capable d'imprimer en 3D. Un modèle de cage thoracique existant a été modifié et imprimé en éléments séparés, comprenant des parties osseuses (cage thoracique, sternum et clavicules), des articulations souples, la peau, le cœur et les poumons, puis assemblé. Le coût total d'impression était de 180 \$CAN. Les recherches

The views expressed in this article are that of the authors and are not an official position of the organizations we are affiliated with.

V. Brannan

vb6161@mun.ca

1 Department of Emergency Medicine, Faculty of Medicine, Memorial University of Newfoundland, 300 Prince Philip Drive, St John's, NL A1B 3V6, Canada

2 Department of Emergency Medicine, University of Calgary, Calgary, AB, Canada

3 Canada Research Chair in HealthCare Simulation, maxSIMhealth Collaborative, Faculty of Health Sciences, Ontario Tech University, Oshawa, ON, Canada

4 Department of Emergency Medicine, Memorial University of Newfoundland, St John's, NL, Canada 
futures se concentreront sur l'incorporation de la capacité du modèle à simuler d'autres procédures HALO et à l'évaluer en tant que complément de formation

\section{Background}

Injuries secondary to trauma are the leading cause of morbidity and mortality for Canadians between the ages 1 and 44 [1]. As a result, emergency medicine (EM) physicians must be competent in performing lifesaving interventions such as tube thoracostomy, surgical airway, thoracotomy, and pericardiocentesis. However, these are all high-acuity low-occurrence procedures where maintaining competence is challenging and lack of provider experience is associated with poor outcomes and life-threatening complications $[2$, 3].

Simulation-based medical education is well established as an effective training approach $[2,4,5]$. Current SBME trainers for high-acuity low-occurrence procedures include commercially manufactured mannequins, animal models, and non-animal models but each have their own associated issues [2, 4-6]. Commercial mannequins, while anatomically accurate and often multifunctional, are costly [6]. These simulators are not a realistic option for many learners, as they are not readily available in smaller or rural centers. Animal and non-animal models can be cheaper but there are issues with tissue realism, landmarking and potential ethical concerns [7].

Three-dimensional (3D)-printed models have the potential to be practical alternative for instruction of high-acuity low-occurrence procedures and avoid barriers such as high cost, issues with availability in smaller centers and lack of realism. A number of trainers have been developed through the use of 3D-printing technology in various medical specialties, however, no published literature has been found on validated 3D-printed trainers for high-acuity low-occurrence procedures [8].

\section{Purpose}

Our aim was to design and manufacture a novel, low-cost, 3D-printed thorax trainer for use in teaching high-acuity low-occurrence procedures. This report describes the development of the thorax trainer and implications for future clinical training and research.

\section{Description of innovation}

Development of the model was through collaborative efforts of a team involving engineers, designers, and clinicians. The initial design was based on an existing ribcage model [9]. This model was separated into five elements using Autodesk ${ }^{\circledR}$ Meshmixer (Version 3.5, Autodesk, Inc 2020) which were printed individually due to printer size constraints. The model was then assembled from the component parts.

The bone portion of the thorax included the ribcage, a sternum, two clavicles, and flexible spine. The ribs, sternum and clavicles were printed as separate pieces using ivorycolored polylactic acid (PLA) and soluble polyvinyl alcohol (PVA) support. At areas in which no flexibility was required, such as between the sternum and collar bones, separate parts were bonded together using glue. The printer used for these sections was the Ultimaker ${ }^{\mathbb{Q}} 3$ 3D Printer.

In areas requiring flexibility, such as between the ribs and sternum, flexible joints were created as single units using thermoplastic elastomer (TPE) 90A as well as PVA. Ribs requiring flexibility were fixed to the sternum and spine using these. The printer used for these joints was the Airwolf $^{\oplus}$ Axiom Dual Direct Drive 3D Printer.

The skin for the thorax was created as a large overlaying portion with a small cut-out replaceable skin section. This allowed repeated use of the model while minimizing waste and associated cost as the large skin overlay was not manipulated during practice. The outer skin had three layers: the top skin layer was made of SmoothOn Ecoflex 00-30, the middle SmoothOn's expanding silicone foam SomaFoma15, and the innermost layer SmoothOn Dragon Skin 10. The skin was made by pouring each successive layer onto a flat surface and then cutting to an appropriate size. The smaller, replaceable skin pieces had an identical composition. Figure 1 shows the completed model design with and without the overlaying skin piece.

This early prototype integrated an anatomically realistic 3D-printed heart and functional "lungs", created using plastic tubing as the "trachea" and two punching balloons for the lungs, inflatable using a bag valve mask setup. These additional materials cost approximately $\$ 10$ and will be refined on future models. The entire model sits on a stand that was designed using Autodesk Fusion 360 on the Ultimaker ${ }^{\circ} 3$ 3D Printer using PLA.

The total cost of the model design was approximately $\$ 180$ CAD. A summary of the equipment, supplies and cost breakdown for each part is included in table form as part of the Online Supplement. 


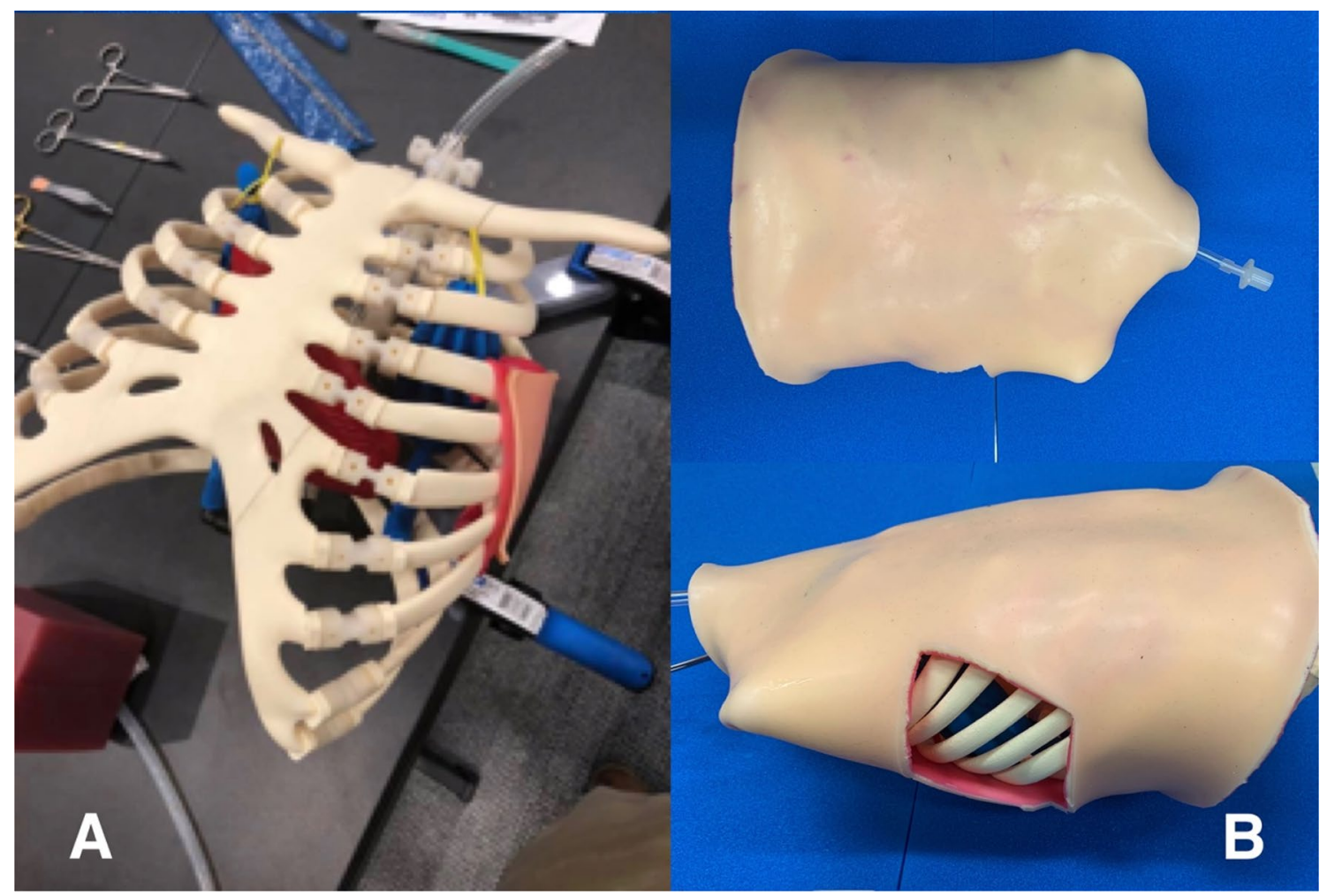

Fig. 1 a 3D-printed thorax model without overlaying skin. Replaceable skin section is visible on left side of thorax. b Complete model with overlaying skin attached (replaceable skin site removed on the lower B image)

\section{Discussion}

Our team created the first documented thorax task trainer for high-acuity low-occurrence procedures using 3D-printing technology. This model is cost-effective, reproducible and reusable, avoiding many of the documented pitfalls of current simulators.

Materials used to build our model were approximately $\$ 180$ CAD. While this price does not include the initial cost of the 3D-printer itself or its design, it remains significantly cheaper than commercial models. As this technology becomes more readily available and open templates are developed, the price of individual units will drop dramatically.

This novel 3D-printed thorax has the potential to be used for simulated learning in rural and remote areas. With the advent of a 3D-printed model that can be reproduced at a low cost, the accessibility of training devices is improved. As well, remote training via tele-simulation has been noted to be an acceptable alternative to in person learning. In one study, researchers compared the educational efficacy of remote instruction to face-to-face instruction, finding that remote procedural skills training produced similarly high levels of satisfaction with learning [10]. A model that can be printed remotely and used through tele-simulation is a potential solution to existing inaccessibility issues.

The 3D-printed thorax task trainer described in this study was an initial prototype and will undergo further development. Brief evaluation surveys were completed by local content experts following use of the model and results indicated that 12 of 14 areas evaluated for realism scored above the cutoff designated as adequate, with the overlaying skin being highlighted as the primary issue. Recommendations to improve the texture and the addition of a fascia layer between ribs to simulate a dissection plane were suggested. Overall, the initial trainer was evaluated as having a global rating of 4.3 out of 5 and all respondents agreed that the trainer required minor to no modifications before use as a teaching adjunct. In addition to improvements regarding tube thoracostomy training, future designs will incorporate more high-acuity low-occurrence procedures into our thorax task trainer. Once updated, a validation study of the model will occur as well as a pre- and post-procedure evaluation to assess utility in developing and maintaining competency for medical learners and physicians. 


\section{Summary}

Using 3D-printing technology, a cost-effective, 3D-printed thorax model was developed for the instruction of tube thoracostomy. This model will help overcome barriers to accessible simulation-based instruction of high-acuity lowoccurrence procedures. Further research is warranted to establish its value as a learning adjunct in simulation.

Supplementary Information The online version contains supplementary material available at https://doi.org/10.1007/s43678-021-00102-1.

Funding Funding for the design of the model was provided by Atlantic Canada Opportunities Agency Business Development Program to Adam Dubrowski. Research and evaluation work was supported by a Summer Undergraduate Research Award from Memorial University of Newfoundland awarded to Victoria Brannan, supervised by Dr. Michael Parsons.

\section{Compliance with ethical standard}

Conflict of Interest The authors declare that they have no conflicts of interest.

\section{References}

1. Fleet R, Archambault P, Plant J, Poitras J. Access to emergency care in rural canada: should we be concerned? CJEM 2013;15(4):191-193. https://www.ncbi.nlm.nih.gov/pubmed/ 23777988. https://doi.org/10.2310/8000.121008. Accessed 29 Apr 2019.

2. Léger A, Ghazali A, Petitpas F, Guéchi Y, Boureau-Voultoury A, Oriot D. Impact of simulation-based training in surgical chest tube insertion on a model of traumatic pneumothorax. Adv Simul (Lond, Engl) 2016;1(1):21. https://www.ncbi.nlm.nih.gov/ pubmed/29449990. https://doi.org/10.1186/s41077-016-0021-2. Accessed 29 Apr 2019.
3. Alrahbi R, Easton R, Bendinelli C, Enninghorst N, Sisak K, Balogh ZJ. Intercostal catheter insertion: are we really doing well? ANZ J Surg 2012;82(6):392-394. https://onlinelibrary.wiley.com/ doi/abs/10.1111/j.1445-2197.2012.06093.x. Accessed 29 Apr 2019.

4. Al-Qadhi S, Pirie J, Constas N, Corrin M, Ali M. An innovative pediatric chest tube insertion task trainer simulation: a technical report and pilot study. Simul Healthe J Soc Simul Healthc 2014;9(5):319-324. https://www.ncbi.nlm.nih.gov/pubmed/24787 558. https://doi.org/10.1097/SIH.0000000000000033. Accessed 29 Apr 2019.

5. Gupta AO, Ramasethu J. An innovative nonanimal simulation trainer for chest tube insertion in neonates. Pediatrics 2014;134(3):e798-e805. https://www.ncbi.nlm.nih.gov/pubmed/ 25092944. https://doi.org/10.1542/peds.2014-0753. Accessed 29 Apr 2019.

6. Ali J, Sorvari A, Pandya A. Teaching emergency surgical skills for trauma resuscitation-mechanical simulator versus animal model. ISRNEmerg Med. 2012;2012:1-6. https://doi.org/10.5402/2012/ 259864.

7. Ching JA, Wachtel TL. A simple device to teach tube thoracostomy. J Trauma Injury Infect Crit Care 2011;70(6):1564-1567. http://ovidsp.ovid.com/ovidweb.cgi?T $=$ JS \&NEWS $=$ n\&CSC $=$ $\mathrm{Y} \& \mathrm{PAGE}=$ fulltext $\& \mathrm{D}=\mathrm{ovft} \& \mathrm{AN}=00005373-201106000-00040$. https://doi.org/10.1097/TA.0b013e318213f5bc. Accessed 29 Apr 2019.

8. Bartellas M, Ryan S, Doucet G, Murphy D, Turner J. Threedimensional printing of a hemorrhagic cervical cancer model for postgraduate gynecological training. Cureus. 2017;9(1):e950. https://www.ncbi.nlm.nih.gov/pubmed/28168128. https://doi.org/ 10.7759/cureus.950. Accessed 29 Apr 2019.

9. Rodriquez A. Thingiverse [Internet]. Maker Bot; 2016. Available from: https://www.thingiverse.com/thing:1543880/files. Cited 6 Aug 2020. Accessed 29 Apr 2019.

10. Jewer J, Parsons MH, Dunne C, Smith A, Dubrowski A. Evaluation of mobile tele-simulation to train rural and remote practitioners on high-acuity low occurrence procedures: pilot randomized controlled trial. J Med Internet Res. 2019;21(8):e14587. https:// doi.org/10.2196/14587. 